\title{
Fuzzy Cube Granule Structure for Image Segmentation
}

\author{
Hongbing Liu, Chunhua Liu, Chang-an Wu and Jun Huang \\ School of Computer and Information Technology, Xinyang Normal University \\ Xinyang 464000, Henan Province, China \\ liuhbing@126.com
}

\begin{abstract}
Fuzzy Cube Granule Structure (FCGS) for image segmentation is proposed in the paper. Firstly, the atomic cube granule is represented as the vector including the $\mathrm{YCbCr}$ values of pixel of color image and radii 0 . Secondly, the join operation between two cube granules is designed to obtain the larger cube granule. Thirdly, the FCGS is formed by the fuzzy inclusion measure defined by join operation and the user-defined granularity threshold $\rho$. Global Consistency Error (GCE), Variation of Information (VI), Rand Index (RI) are used to evaluate the segmentations. Images selected from BSD300 are used to verify the feasibility of FCGS.
\end{abstract}

Keywords: Cube granule, join operation, fuzzy cube granule structure, image segmentation

\section{Introduction}

Granular computing $(\mathrm{GrC})$ concerns the processing of complex information entities called information granules, which arise in the process of data abstraction and derivation of knowledge from information or data [1,2]. In the philosophical sense, granular computing can describe a way of thinking that relies on the human ability to recognize the real world under various levels of granularity in order to abstract and consider only those things that serve a specific interest and to switch among different granularities. By focusing on different levels of granularity, one can obtain different levels of knowledge, as well as a greater understanding of the inherent knowledge structure. Granular computing is thus essential in human problem solving and hence has a very significant impact on the design and implementation of intelligent systems, such as classification problems [3-5].

In computer vision, image segmentation is the process of partitioning a digital image into multiple segments. The goal of segmentation is to simplify and/or change the representation of an image into something that is more meaningful and easier to analyze [6]. Image segmentation is typically used to locate objects and boundaries in images. More precisely, image segmentation is the process of assigning a label to every pixel in an image such that pixels with the same label share certain visual characteristics. The result of image segmentation is a set of segments that collectively cover the entire image, or a set of contours extracted from the image. Each of the pixels in a region is similar with respect to some characteristic or computed property, such as color, intensity, or texture. Adjacent regions are significantly different with respect to the same characteristic [6]. When applied to a stack of images, the resulting contours after image segmentation can be used to create 3D reconstructions with the help of interpolation algorithms. The practical applications of image segmentation included content-based image retrieval [7], machine vision [8], medical imaging [9], and object detection [10].

The present work forms the fuzzy cube granule structure for image segmentation, which is a clustering method. For the image I, we convert the RGB values into the $\mathrm{YCbCr}$ 
color space since humans are more sensitive to illuminance changes. Firstly, $\mathrm{YCbCr}$ values of pixel point are used to represent the atomic cube granule which is inseparable. Secondly, fuzzy inclusion measure is defined by the join cube granule between two cube granules. Thirdly, the user-defined threshold of granularity is used to control the join process, and the data belonging to the same cube granule is composed of the cluster.

The rest of this paper is presented as follows: Fuzzy cube granule structure is described in Section 2. Section 3 demonstrates the comparative experimental results on image segmentation problems. Section 4 summarizes the contribution of our work.

\section{Fuzzy Cube Granule Structures}

For the data set $S=\left\{x_{i} \mid i=1,2, \ldots, n\right\}$ of image segmentation problems in 3-dimensional space induced by the $\mathrm{YCbCr}$ values of pixels, we construct fuzzy cube granule structure in terms of the following steps. Firstly, a granule is represented as a cube, and the size of granule is measured by the granularity induced by the ridge of cube. Secondly, operations between two cube granules are designed to obtain the cube granule with the larger granularity, and the fuzzy inclusion measure between two cube granules is induced by granule and the operated granule. Thirdly, the fuzzy cube granule structure is formed.

\subsection{Representation of Cube Granule}

In reality, the shapes of granules are irregular. In order to study granule, the shapes are represented as the regular forms. In 3-dimensional space induced by $\mathrm{YCbCr}$ values of pixel, the granule is represented as the cube, and the granularity of granule is the half of ridge. So the cube granule is represented as follows.

$$
\boldsymbol{G}=(C, R)
$$

Where $\mathrm{C}$ is the center of cube, and $\mathrm{R}$ is the half of ridge. For the image $\mathrm{I}$, the $\mathrm{YCbCr}$ values of pixel are represented as a atomic cube granule by formula (1).

\subsection{Operations Between Two Granules}

The join process is the key to obtain the larger granules compared with atomic granules. For two cube granules $\boldsymbol{G}_{1}=\left(\boldsymbol{C}_{1}, R_{1}\right)$ and $\boldsymbol{G}_{2}=\left(\boldsymbol{C}_{2}, R_{2}\right)$ in 3-dimensional space, the central vector $\boldsymbol{C}$ of $\boldsymbol{G}$ and the granularity $R$ of the join granule $\boldsymbol{G}=\boldsymbol{G}_{1} \vee \boldsymbol{G}_{2}=(\boldsymbol{C}, R)$ are computed by algorithm1.

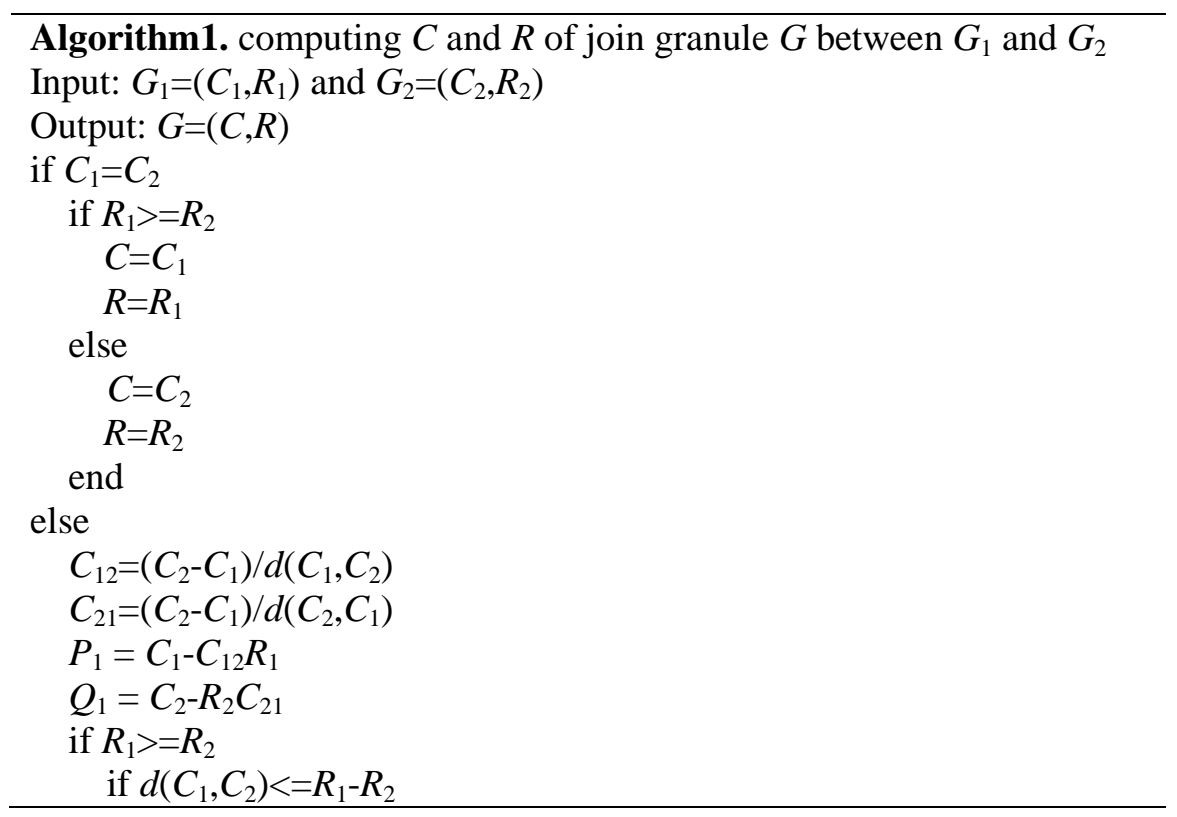




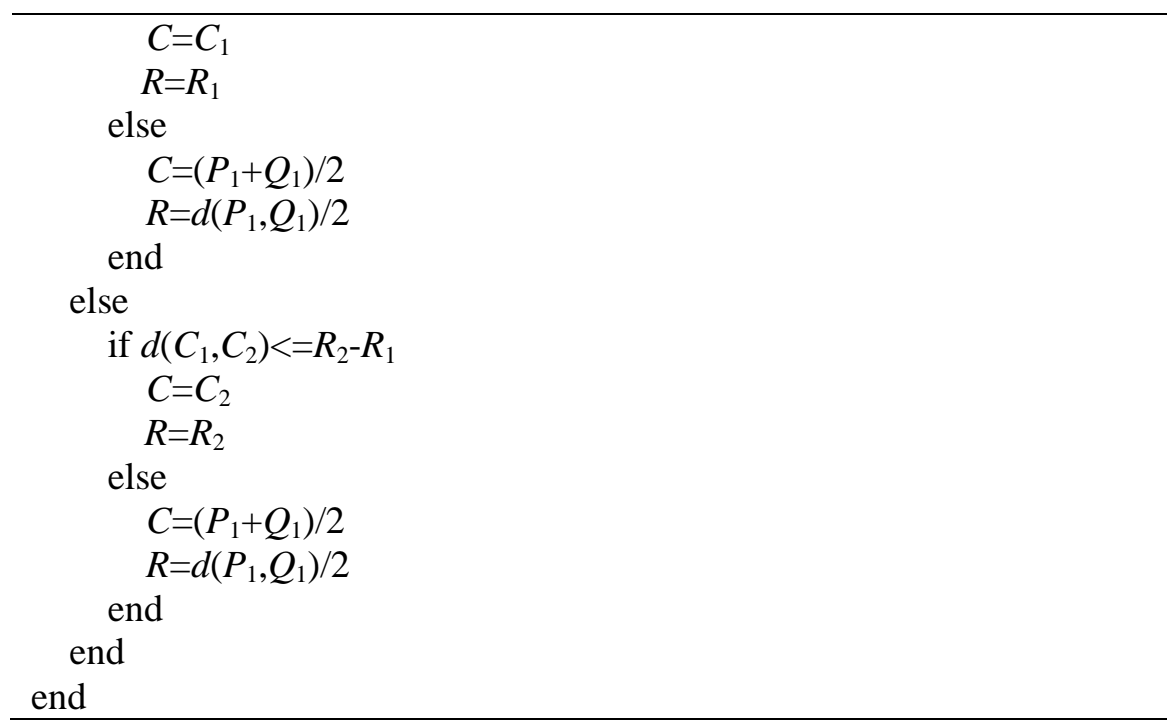

We explain the join process by two squares in 2-dimensional space. For square granule $G_{1}=\left[\begin{array}{lll}0.2 & 0.15 & 0.1\end{array}\right]$ and square granule $G_{2}=\left[\begin{array}{lll}0.08 & 0.25 & 0.06\end{array}\right]$, the join square granule is $G=[0.16,0.1833,0.14]$ shown in Figure 1.

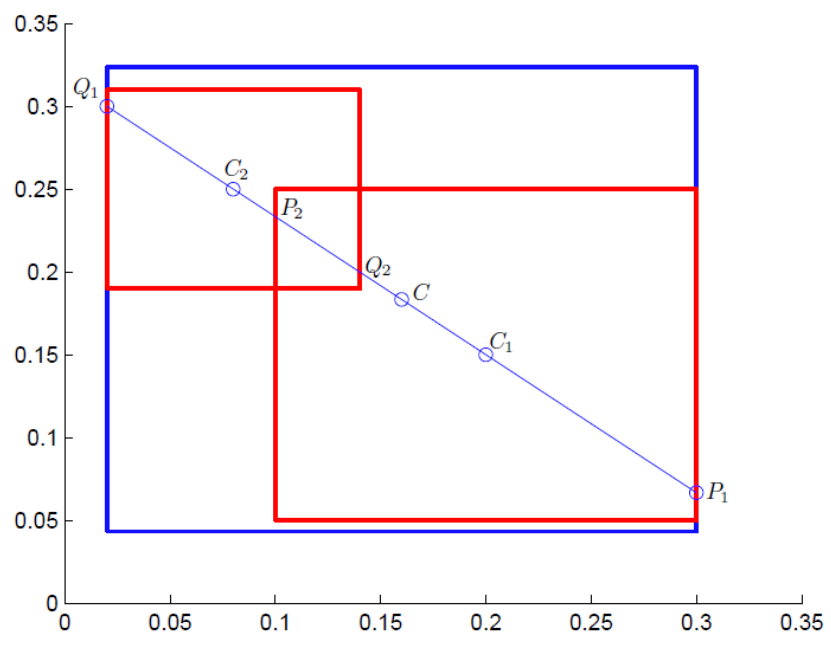
Figure 1. The Join Ssquare Granule of Two Square Granules in 2-
Dimensional Space

From Figure1, $G_{1} \subseteq G_{1} \vee G_{2}$ and $G_{2} \subseteq G_{1} \vee G_{2}$. Namely, the operations between granule $G_{1}$ and granule $G_{2}$ are corresponding to the inclusion relation between granule $G_{1}$ and $G_{2}$.

$$
G_{1} \subseteq G_{2} \Leftrightarrow G_{1} \vee G_{2}=G_{2}, G_{1} \wedge G_{2}=G_{1}
$$

The inclusion measure between two granules is induced by the operations between two granules.

\subsection{Fuzzy Inclusion Measure}

As a subset of data set $S$, the inclusion relation between two cube granules is fuzzy. For example, $G_{1}=[0.1,0.2,0.1]$ and $G_{2}=[0.32,0.2,0.1]$ have the same granularity and different central vector, and $G=[0.4,0.3,0.06]$. The inclusion measure between $G_{1}$ and $G$ are different from the inclusion measure between $G_{2}$ and $G$, especially there is overlap between $G_{2}$ and $G$. The fuzzy inclusion measure is used to measure the fuzzy inclusion relation. In Figure 2, one hand is the difference of the join hypercube granule $G_{1} \vee G=[0.23,0.2433,0.23]$ and the join hypercube granule $G_{2} \vee G=[0.344,0.23,0.13]$, 
the other hand is the difference of the meet hypercube granule $G_{1} \wedge G=\varnothing$ and the meet hypercube granule $G_{2} \wedge G=[0.367,0.27,0.03]$. The fuzzy inclusion measure between $G_{2}$ and $G$ is greater than the fuzzy inclusion measure between $G_{1}$ and $G$.

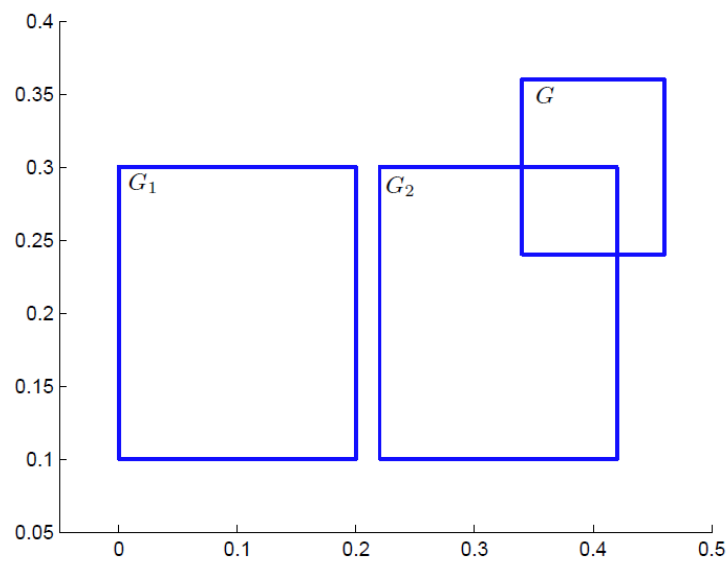

\section{Figure 2. The Different Inclusion Measure between Two Granules}

The join granule and the meet granule are used to measure the fuzzy inclusion relation. The granularity $R$ is used to define the fuzzy inclusion measure.

$$
K\left(G_{1}, G\right)=v(G) / v\left(G_{1} \vee G\right)
$$

Where $v(G)$ is the positive valuation function defined by $\mathrm{V}$ G kaburlasos, which can be the linear function or nonlinear function [3-5].

\subsection{Fuzzy Cube Granule Structure}

In recent years, lattice computing is used in clustering problems [11-13]. For a data set $S=\left\{x_{i} \mid i=1,2, \ldots, n\right\}$, every datum $x_{i}$ is represented as an atomic cube granule which is indivisible. The granule set (GS) is induced by the representation method of granule, the join operation is defined on granule set GS, and used to compound the fuzzy inclusion measure (2). So the FCGS <GS, $K(.,)$.$\rangle is formed by the granule set GS induced by$ representation of granule, and fuzzy inclusion measure $K(. .$,$) between two granules$ induced by operation between two granules.

Suppose the data set $S=\left\{\mathrm{g}_{1}-\mathrm{g}_{5}\right\}$. The clustering process can be described as the following tree structure shown in Figure.3, leafs denote the atomic cube granules, root denotes $G S$ including its child nodes $G_{1}, G_{2}$, and $\mathrm{g}_{3} . G_{1}$ is induced by join operation of child nodes $\mathrm{g}_{1}$ and $\mathrm{g}_{2}, G_{2}$ is the join cube granule of $\mathrm{g}_{4}$ and $\mathrm{g}_{5}, \mathrm{~g}_{3}$ is the atomic cube granule. The whole process of obtaining $G S$ is the bottle up process.

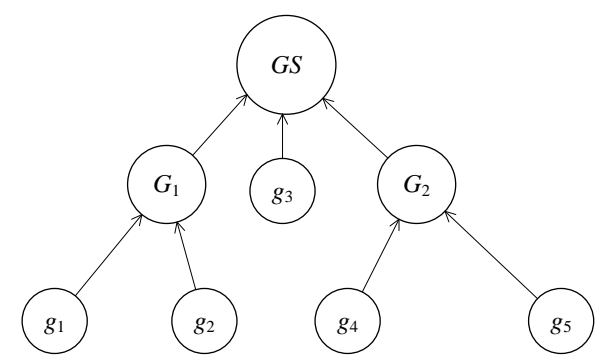

Figure 3. The Clustering Process of Data Set Including 5 Samples

The clustering algorithm of fuzzy cube granule structure is described as algorithm2. 


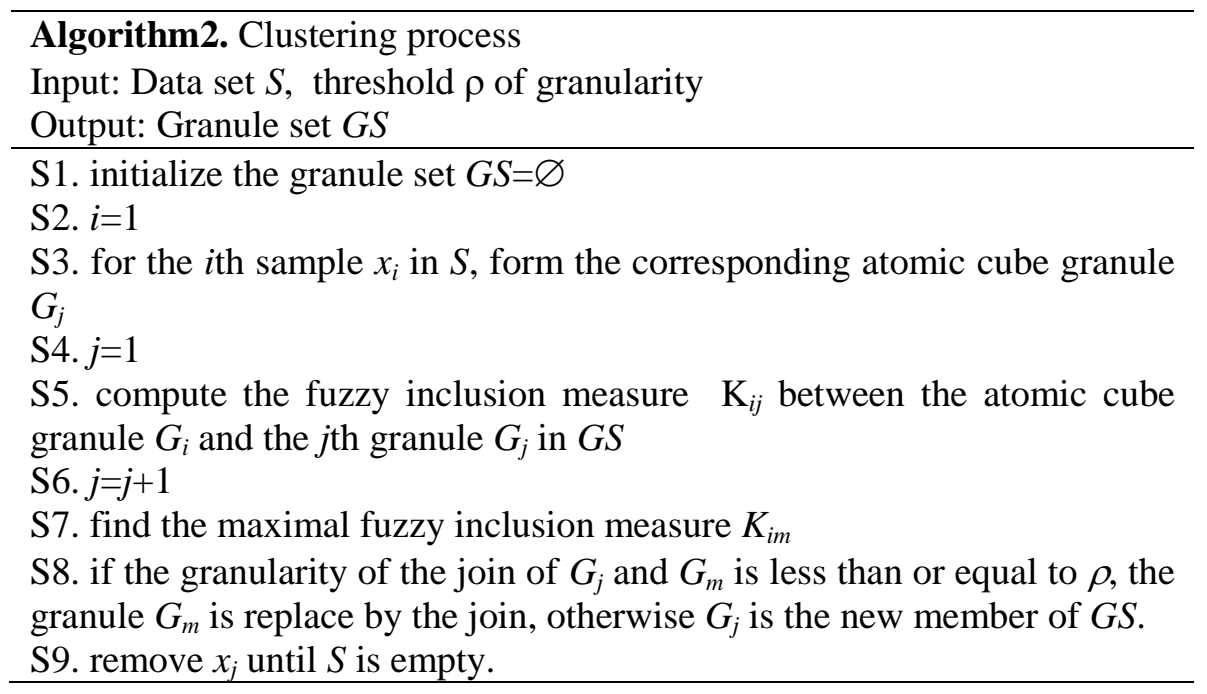

\section{Evaluation of Segmentation}

For the segmentations $S=\left\{S_{1}, S_{2}, \ldots, S_{n}\right\}$ and $S^{\prime}=\left\{S_{1}{ }_{1}, S_{2}{ }_{2}, \ldots, S_{m}{ }_{m}\right\}$ of original color image $I$ with $n_{1}$ rows and $n_{2}$ columns, and $N=\left|S_{1}\right|+\left|S_{2}\right|+\ldots+\left|S_{n}\right|=\left|S_{1}{ }_{1}\right|+\left|S_{2}{ }_{2}\right|+\ldots+\left|S_{m}{ }_{m}\right|$, we evaluate the segmentation by the following aspects.

\subsection{Global Consistency Error}

D. Martin proposed several error measures to quantify the consistency between image segmentations of differing granularity $[14,15]$. Let $S$ and $S$ ' be two segmentations of an image $I=\left(x_{1}, x_{2}, \ldots, x_{N}\right)$ consisting of $N$ pixels. For a given pixel $x_{i}$, consider the classes (segments) that contain $x_{i}$ in $S$ and $S^{\prime}$. We denote these sets of pixels by $C\left(S, x_{i}\right)$ and $C$ $\left(S^{\prime}, x_{i}\right)$, respectively. Local Refinement Error (LRE) is then defined at point $x_{i}$ as:

$\operatorname{LRE}\left(S, S^{\prime}, x_{i}\right)=\left|C\left(S, x_{i}\right)-C\left(S^{\prime}, x_{i}\right)\right| /\left|C\left(S, x_{i}\right)\right|$

Where $C\left(S, x_{i}\right)-C\left(S^{\prime}, x_{i}\right)$ denotes the set differencing operator between sets $C\left(S, x_{i}\right)$ and $C\left(S^{\prime}, x_{i}\right)$. This error measure is not symmetric and encodes a measure of refinement in one direction only. There are two natural ways to combine the LRE at each point into a measure for the entire image. Global Consistency Error (GCE) forces all local refinements to be in the same direction and is defined as:

$\operatorname{GCE}\left(\mathrm{S}, \mathrm{S}^{\prime}\right)=\min \left\{\operatorname{LRE}\left(S, S^{\prime}, x_{1}\right)+\ldots+\operatorname{LRE}\left(S, S^{\prime}, x_{N}\right), \operatorname{LRE}\left(S^{\prime}, S, x_{1}\right)+\ldots+\operatorname{LRE}\left(S, S^{\prime}, x_{N}\right)\right\} / N$

\subsection{Variation of Information}

Work in [16] computes a measure of information content in each of the segmentations. The proposed measure, termed the Variation of Information (VI), is a metric and is related to the conditional entropies between the class label distribution of the segmentations. The measure has several promising properties [16] but its potential for evaluating results on natural images where there is more than one ground-truth clustering is unclear. The VI is computed by the following steps. Firstly, computing the entropies $\operatorname{En}(S)$ and $\operatorname{En}\left(S^{\prime}\right)$ associated with segmentation $\mathrm{S}$ and $\mathrm{S}$ '.

$\mathrm{E}_{\mathrm{n}}(S)=-\left(P(1) \log _{2} P(1)+P(2) \log _{2} P(2)+\ldots+P(n) \log _{2} P(n)\right)$

Where $P(i)=\left|S_{i}\right| / N$

$\mathrm{E}_{\mathrm{n}}\left(S^{\prime}\right)=-\left(P^{\prime}(1) \log _{2} P^{\prime}(1)+P^{\prime}(2) \log _{2} P^{\prime}(2)+\ldots+P^{\prime}(m) \log _{2} P^{\prime}(m)\right)$

Where $P^{\prime}(i)=\left|S^{\prime}\right| / N, \log _{2} 0=0$.

Secondly, computing the mutual information between $\mathrm{S}$ and $\mathrm{S}^{\prime}$

$$
I\left(S, S^{\prime}\right)=\sum_{i=1}^{n} \sum_{j=1}^{m} P(i, j) \log _{2} \frac{P(i, j)}{P(i) P^{\prime}(j)}
$$

Thirdly, computing the VI 


$$
\mathrm{VI}\left(S, S^{\prime}\right)=\mathrm{E}_{\mathrm{n}}(S)+\mathrm{E}_{\mathrm{n}}\left(S^{\prime}\right)-2 \mathrm{I}\left(S, S^{\prime}\right)
$$

\subsection{Rand Index}

Rand Index (RI) was motivated by standard classification problems in which the result of a classification scheme has to be compared to a correct classification [17]. The most common performance measure for this problem calculates the fraction of correctly classified (respectively misclassified) elements to all elements. For Rand, comparing two clusters was just a natural extension of this problem which has a corresponding extension of the performance measure: instead of counting single elements he counts correctly classified pairs of elements. Thus, RI is defined by:

$\mathrm{RI}\left(S, S^{\prime}\right)=2\left(N_{11}+N_{00}\right) /(N(N-1))$

Where $N_{11}$ denotes the numbers of pairs that are in the same cluster under $S$ and $S^{\prime}, N_{00}$ denotes the number of pairs that are in different clusters under $S$ and $S^{\prime}$. RI depends on both the number of clusters and the number of elements, and ranges from 0 to 1 . S and $S$, are identical when RI equals to 1.

\section{Experiments}

We evaluated the effectiveness of our algorithms induced by the FCGS for image segmentation selected from BSD300 (http://www.eecs. berkeley. edu/Research /Projects /CS/vision/bsds/), with an Intel Core i5 PC with $3.2 \mathrm{GHz}$ CPU and 8 GB memory, running Microsoft Win7 and Matlab R2008a.

For the color image 24063 in BSD300, the parameter of granularity are set from 0.5 to 0.2 with the step 0.02 , our purpose is to find the parameter $\rho$ who makes the minimal GCE and VI, and the maximal RI. GCE, VI, and RI of image 24063 by GrCC are listed in Table 1. From the Table, we can see GrCC achieved the minimal GCE (0.0506), the minimal VI (0.7763), and the maximal RI (0.9115). The relationships between parameter and $\rho$ are shown in Figure.4, from the Figure, we can see GCE and VI reached the lowest points 0.0506 and 0.7763 , and RI reached the highest point 0.9115 when parameter $\rho=0.22$. Figure. 5 includes the original image and the boundary of human segmentation.

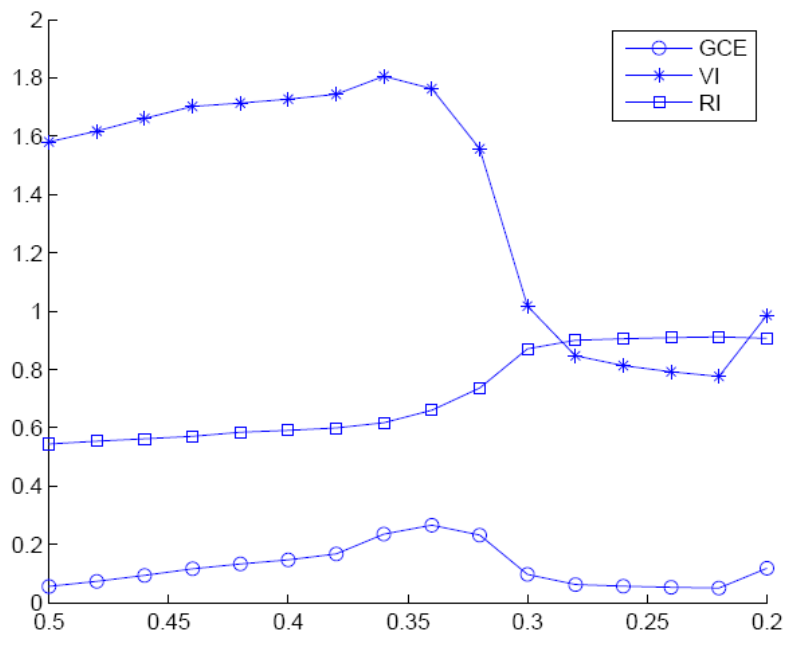

Figure 4. The Relationships between Parameter $\rho$ and GCE, VI, and RI 
Table 1. The Performance of Segmentations for Image 24063 in BSD300

\begin{tabular}{|c|r|c|c|c|}
\hline$\rho$ & \#cls & GCE & VI & RI \\
\hline 0.5000 & 2 & 0.0568 & 1.5798 & 0.5448 \\
0.4800 & 2 & 0.0739 & 1.6174 & 0.5541 \\
0.4600 & 2 & 0.0941 & 1.6604 & 0.5623 \\
0.4400 & 2 & 0.1169 & 1.7019 & 0.5709 \\
0.4200 & 2 & 0.1332 & 1.7132 & 0.5846 \\
0.4000 & 2 & 0.1475 & 1.7264 & 0.5916 \\
0.3800 & 2 & 0.1679 & 1.7432 & 0.5996 \\
0.3600 & 3 & 0.2358 & 1.8050 & 0.6173 \\
0.3400 & 3 & 0.2663 & 1.7627 & 0.6604 \\
0.3200 & 3 & 0.2322 & 1.5562 & 0.7366 \\
0.3000 & 3 & 0.0973 & 1.0175 & 0.8708 \\
0.2800 & 3 & 0.0632 & 0.8473 & 0.9002 \\
0.2600 & 3 & 0.0570 & 0.8133 & 0.9056 \\
0.2400 & 3 & 0.0531 & 0.7918 & 0.9089 \\
0.2200 & 3 & 0.0506 & 0.7763 & 0.9115 \\
0.2000 & 4 & 0.1187 & 0.9859 & 0.9065 \\
\hline
\end{tabular}
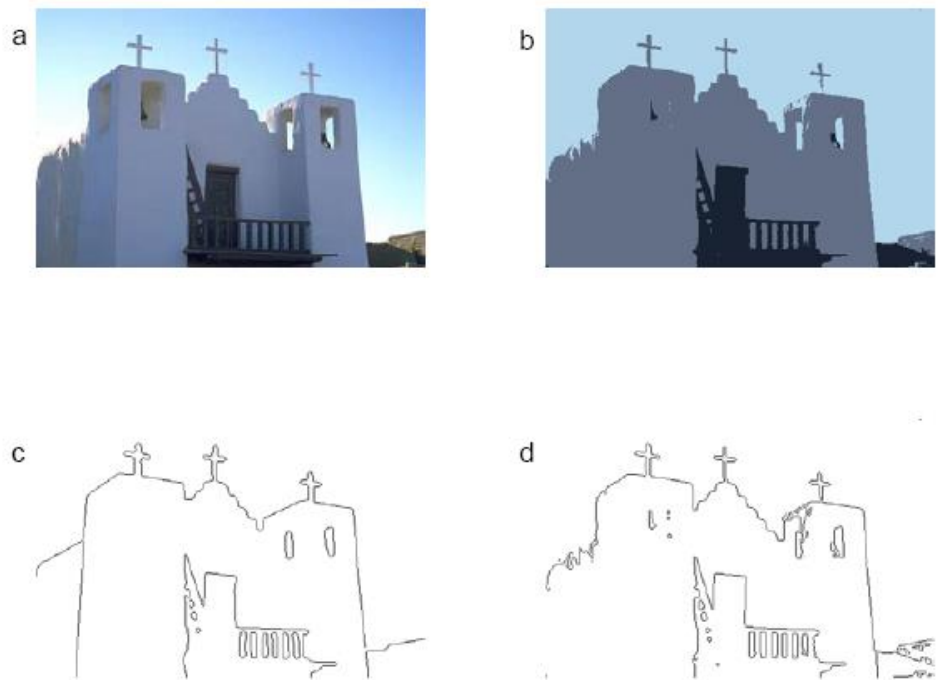

Figure 5. Image and its Segmentation Image, Boundary by Human, and Boundary by FCGS ( $\rho=0.22)$. (a) is the Original Image, (b) the Segmentation Image, (c) Boundary by Human, (d) Boundary by FCGS 

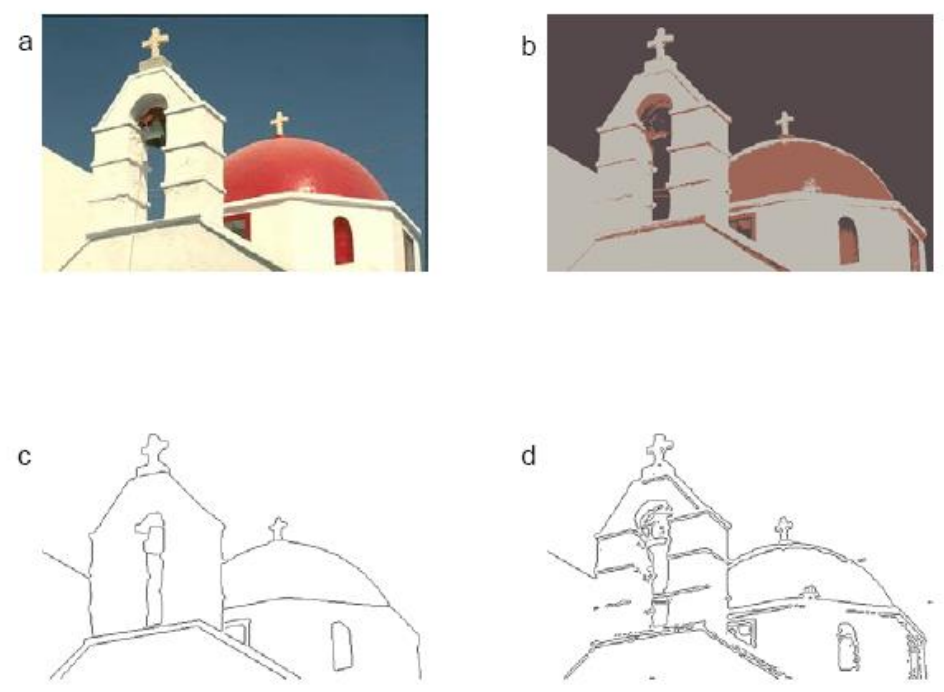

Figure 6. Image (118035) and its Segmentation Image, Boundary by Human, and Boundary by FCGS $(\rho=0.28)$. (a) is the Original Image, (b) the Segmentation Image, (c) Boundary by Human, (d) Boundary by FCGS

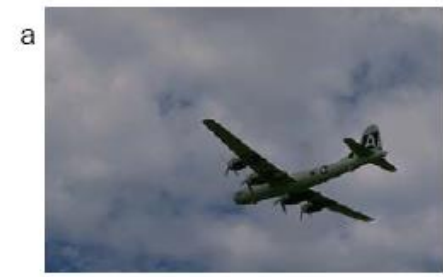

C

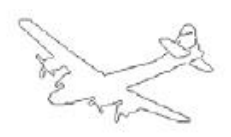

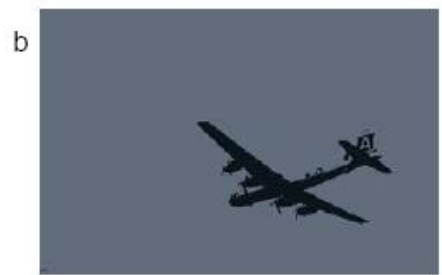

d

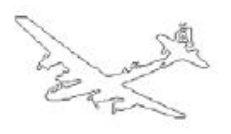

Figure 7. Image (3069) and its Segmentation Image, Boundary by Human, and Boundary by FCGS $(\rho=0.34)$. (a) is the Original Image, (b) the Segmentation Image, (c) Boundary by Human, (d) Boundary by FCGS

\section{Conclusion}

The representation of granule, the join operation between two granules, the fuzzy inclusion measures between two granules is discussed in details. The fuzzy granule algebraic structure is induced by the granule set, operations between two granules, and fuzzy inclusion measure between two granules, and used to form the granular computing clustering algorithm for image segmentation. The experimental results showed the feasibility of FCGS for image segmentation. 


\section{Acknowledgments}

This work was supported in part by the Natural Science Foundation of China (Grant no. $61170202,61402393)$

\section{References}

[1] L. A. Zadeh, "Fuzzy Sets and Information Granulation," North Holland Publishing, (1979).

[2] L. A. Zadeh, "Toward a theory of fuzzy information granulation and its centrality in human reasoning and fuzzy logic," Fuzzy Sets and Systems, vol.90, (1997).

[3] V. G. Kaburlasos, I. N. Athanasiadis and P. A. Mitkas, "Fuzzy Lattice Reasoning (FLR) classifier and its application for ambient ozone estimation," International Journal of Approximate Reasoning, vol.45, no.1, (2007).

[4] V. G. Kaburlasos and S. E. Papadakis, "A granular extension of the Fuzzy-ARTMAP (FAM) neural classifier based on Fuzzy Lattice Reasoning (FLR)," Neurocomputing, vol.72 no.10-12, (2009).

[5] V. G. Kaburlasos and V. Petridis, "Fuzzy Lattice Neurocomputing (FLN) models," Neural Networks, vol. 13 no. 10, (2000).

[6] L. G. Shapiro and G. C. Stockman, "Computer Vision,” New Jersey, Prentice-Hall, (2001).

[7] M. S. Lew, N. Sebe, C. Djeraba and R. Jain, "Content based multimedia information retrieval: State of the art and challenges," ACM Transactions on Multimedia Computing, Communications and Applications, vol. 2 no.1, (2006).

[8] F. D. Turek, "Machine vision fundamentals, How to make robots see," NASA Tech Briefs, vol.35 no.6, (2011).

[9] D. L. Pham, C. Xu and J. L. Prince, "Current methods in medical image segmentation," Annual Review of Biomedical Engineering, vol.2, (2000).

[10] F. Fleuret, J. Berclaz, R. Lengagne and P. Fua. "Multi-camera people tracking with a probabilistic occupancy map," IEEE Transactions on Pattern Analysis and Machine Intelligence, vol.30 no.2, (2008).

[11] M. Graña, I. Villaverde, J. O. Maldonado and C. Hernandez, "Two lattice computing approaches for the unsupervised segmentation of hyperspectral images," Neurocomputing, vol.72 no.10-12, (2009).

[12] M. Graña, A. M. Savio, M. García-Sebastián and E. Fernandez, "A lattice computing approach for on-line fMRI analysis," Image and Vision Computing, vol.28 no.7, (2010).

[13] V. Syrris and V. Petridis, "A lattice-based neuro-computing methodology for real-time human action recognition," Information Sciences, vol.181 no.10, (2011).

[14] D. Martin, "An empirical approach to grouping and segmentation," Ph.D. dissertation, University of California, Berkeley, (2002).

[15] D. R. Martin, C. Fowlkes and J. Malik, "Learning to detect natural image boundaries using local brightness, color, and texture cues," IEEE Transaction Pattern Analysis Machine Intelligence, vol.26 no.5, (2004).

[16] M. Meilă, "Comparing clusterings an information based distance," Journal of Multivariate Analysis, vol.98 no.5, (2007).

[17] W. M. Randm, "Objective criteria for the evaluation of clustering methods," Journal of the American Statistical Association, vol.66 no.336, (1971). 
International Journal of Multimedia and Ubiquitous Engineering

Vol.10, No.6 (2015) 